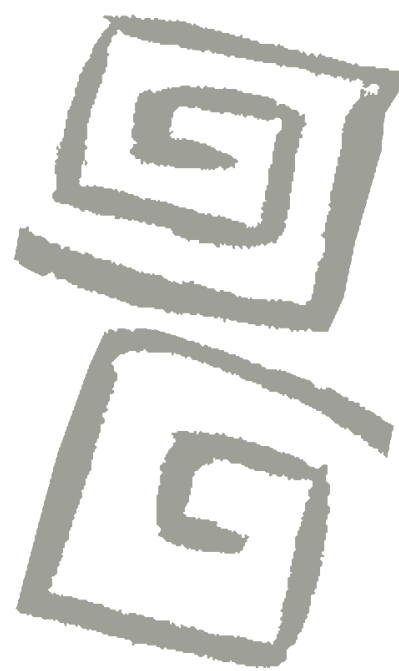

\title{
Estudio cualitativo sobre prácticas de riesgo y de cuidado en escenarios nocturnos de presencia masiva de la Ciudad Autónoma de Buenos Aires
}

\author{
Qualitative study on risk and care practices in nightlife \\ settings with massive presence of people in Buenos \\ Aires City
}

Vanina Schmidt ${ }^{1}$, Florencia Martucci², Gabriela Di Puglia ${ }^{3}$, Ornella Lo Giusto ${ }^{4}$, Ignacio Rijana ${ }^{5}$, Analia Alvarez Iturain ${ }^{6}$

${ }^{1}$ Doctora en Psicología. Investigadora Independiente del Consejo Nacional de Investigaciones Científicas y Técnicas. Profesora titular regular, Universidad Abierta Interamericana, Ciudad Autónoma de Buenos Aires, Argentina. $\bowtie$ (iD)

${ }^{2}$ Licenciada en Psicología. Investigadora, Universidad de Buenos Aires, Ciudad Autónoma de Buenos Aires, Argentina. $\square$ (iD)

${ }^{3}$ Licenciada en Psicología. Becaria, Ministerio de Salud, Gobierno de la Ciudad de Buenos Aires. Profesora Interina e investigadora, Universidad Abierta Interamericana, Ciudad Autónoma de Buenos Aires, Argentina. $\square$ (iD)

${ }^{4}$ Licenciada en Psicología. Ayudante de Trabajos Prácticos, Universidad de Buenos Aires. Investigadora, Universidad Abierta Interamericana. Ciudad Autónoma de Buenos Aires, Argentina. $\square$ (iD)

${ }^{5}$ Licenciado en Psicología. Profesor e investigador, Universidad de Buenos Aires. Investigador, Universidad Abierta Interamericana.

Ciudad Autónoma de Buenos Aires, Argentina. $\square$ (iD

${ }^{6}$ Licenciada en Psicología. Ayudante de Trabajos Prácticos, Universidad de Buenos Aires. Profesora Interina e investigadora, Universidad Abierta Interamericana. Ciudad Autónoma de Buenos Aires, Argentina. $\square$ (iD
RESUMEN El objetivo del presente estudio fue indagar las prácticas de cuidado y de riesgo que se despliegan en escenarios nocturnos de presencia masiva de la Ciudad Autónoma de Buenos Aires. Asimismo, se indagaron los motivos percibidos para el consumo de sustancias psicoactivas. Para ello se entrevistó a 16 jóvenes de entre 21 a 35 años de edad. Los datos fueron analizados siguiendo la propuesta de la teoría fundamentada y con el soporte del programa Atlas.Ti. Se analizaron prácticas individuales, prácticas grupales, y a nivel del entorno. Entre las prácticas de riesgo se mencionaron con frecuencia la disponibilidad de las sustancias, la ingesta excesiva y las mezclas. Respecto de las prácticas de cuidado, se destacó el valor del grupo al mantenerse todos juntos y brindar ayuda a sus miembros. Se discuten las implicancias prácticas de estos resultados, y se presentan las limitaciones y líneas futuras de investigación.

PALABRAS CLAVES Sustancias Psicoactivas; Percepción Social; Riesgo; Argentina.

ABSTRACT The aim of the present study was to investigate care and risk practices carried out in nightlife settings with massive presence of people in the Autonomous City of Buenos Aires. In addition to this, the perceived reasons behind the consumption of psychoactive substances were investigated. For this purpose, 16 young people between 21 and 35 years of age were interviewed. The data was analyzed following the approach proposed in the grounded theory and using the Atlas.Ti program. Practices were studied at individual, group and environmental levels. Among the risk practices, substance availability, excessive intake and substance mixing were frequently mentioned. Regarding care practices, the value of the group was highlighted by keeping together and providing help to its members. Practical implications of these results are discussed, and limitations and future lines of research are presented.

KEY WORDS Psychoactive Substances; Social Perception; Risks; Argentina. 


\section{INTRODUCCIÓN}

En las sociedades actuales los espacios destinados al ocio nocturno se han transformado en un lugar clave del contexto recreativo juvenil(1). El objetivo principal del presente estudio fue indagar desde la percepción de los jóvenes las prácticas de riesgo y cuidado que tienen lugar en escenarios nocturnos de presencia masiva de la Ciudad Autónoma de Buenos Aires. Asimismo, como objetivo secundario, se indagaron los motivos para consumir sustancias psicoactivas en estos escenarios. Se entiende por factores de riesgocuidado los estímulos o situaciones sociales, familiares y personales que determinan una mayor o menor vulnerabilidad respecto del uso de drogas ${ }^{(2)}$. En el presente estudio, el foco está ubicado en las percepciones que los protagonistas de los mencionados escenarios poseen respecto de los riesgos y cuidados. Siguiendo el planteo de Sánchez Antelo y Mendes-Diz ${ }^{(3)}$ se concibe la "percepción de los riesgos" como un producto social regulado por las prácticas e interacciones sociales, al tiempo que manifiesta los sentidos asignados a esas prácticas que, si bien se consideran dañinas, también se evalúan como deseables/ placenteras, lo que evidencia diversos grados de aceptabilidad frente a sus consecuencias. Güelman ${ }^{(4)}$ define las prácticas de cuidado en referencia a aquellas estrategias dirigidas a prevenir o minimizar las consecuencias negativas asociadas al uso de drogas.

En la presente investigación, se incluye una variedad de escenarios nocturnos (bar, pub, fiesta electrónica y boliche) que ofrece la Ciudad Autónoma de Buenos Aires. Según el Registro de Locales Bailables ${ }^{(5)}$, en esta ciudad hay 160 locales inscriptos de baile, bares, restaurantes u otros rubros, cuya actividad complementaria es el baile. Estos espacios quedan definidos y diferenciados por una serie de elementos: el género musical, la estética de quienes asisten y del lugar, las sustancias que se consumen, los modos de relacionarse, las edades de los concurrentes, la zona geográfica, el costo de la entrada, entre otros.
Durante décadas, los boliches fueron las actividades recreativas nocturnas más frecuentes entre los jóvenes de clase media en la Ciudad de Buenos Aires, a partir de la década de 1990, comienza la era dance-electrónica. Las diferencias entre ambos escenarios son notables. A los boliches asiste una amplia variedad de jóvenes en términos de edad, situación laboral y educativa, y nivel socioeconómico; mientras que las fiestas electrónicas fueron inicialmente el lugar privilegiado para un selecto y reducido grupo perteneciente a sectores medio-altos y altos y, luego, con el correr de los años, se masificaron y atrajeron a un público urbano joven más amplio, aunque siguen siendo de alta inserción en el sistema educativo y el mundo laboral, y poseen mayormente cobertura social ${ }^{(6)}$. En los boliches se encuentran ritmos variados (rock, marcha, pop, melódico, cumbia, etc.) mientras que, en las fiestas, la música electrónica no convive con ningún otro estilo musical. La forma de difusión de cada tipo de evento también cambia. Hay tarjeteros y jóvenes destinados a las relaciones públicas que promocionan los boliches; en cambio, en el caso de las fiestas electrónicas, la publicidad no está tan disponible ni es tan clara, y sigue la lógica de ser un espacio solo para entendidos. En Las fiestas electrónicas no hay personal encargado de seleccionar quiénes ingresan y quiénes serán excluidos, sino que la selección está dada por el alto costo de la entrada o la dificultad para conseguir la información. Las drogas de síntesis y el éxtasis como su máximo representante son elementos significativos en las fiestas electrónicas; mientras que, en los boliches, las sustancias de consumo son más variadas. Los escenarios nocturnos de música electrónica generan más confianza en los participantes respecto de las sustancias que se consumen y a la regulación de los efectos no deseados. A su vez, la gente percibe que en dichos escenarios hay menor cantidad de episodios violentos ${ }^{(1,6,7)}$

Otros espacios han conquistado la escena nocturna como opción recreativa entre los jóvenes en la Ciudad de Buenos Aires. Se trata de los bares y pubs. En ambos casos se ofrece una gran variedad de bebidas 
alcohólicas, como cócteles, tragos, cerveza, vino, entre otros, y predominan géneros musicales de los más variados (pop, rock, cumbia, trap, etc.). En los bares, puede haber música en vivo e, incluso, pista de baile. En los últimos años, hubo una proliferaron los pubs dedicados a la venta de cerveza artesanal, con una estética más moderna que la de los bares, que se han transformado en uno de los espacios preferidos por los adultos jóvenes de clase media. Estas cervecerías artesanales trabajan pocas horas (suelen abrir a las siete de la tarde, y solo los fines de semana se extienden hasta la madrugada), a diferencia de los bares que pueden permanecer abiertos todo el día ${ }^{(8)}$.

En todos estos espacios recreativos se inscriben las prácticas que forman parte del complejo entramado de la categoría de lo juvenil en nuestras sociedades. Y también en estos espacios, el uso de drogas tanto legales como ilegales representa una práctica cada vez más esperable y menos reflexiva ${ }^{(1)}$. Las sustancias pasan a ser interpretadas como facilitadoras de la diversión, enfatizando la conexión con los otros y un sentirse bien con uno mismo. El propio término drogas recreativas hace alusión a su contexto de consumo, vinculado al entretenimiento del fin de semana, a la música, al baile, a los eventos o megafiestas y al ambiente nocturno ${ }^{(9)}$.

En lo que respecta al marco normativo vigente sobre la legalidad de las sustancias se encuentra una coexistencia de criterios diversos que no confluyen en una perspectiva homogénea. Por un lado, se encuentra vigente la Ley 23737 de Tenencia y tráfico de estupefacientes, del año 1989, que establece la penalización de la tenencia, como así también el cultivo y comercialización (entre otras cuestiones). En el año 2009, el conocido "Fallo Arriola" de la Corte Suprema de Justicia instala jurisprudencia al respecto, ya que declara inconstitucional el Artículo 14 de la Ley 23737, argumentando que penalizar el consumo personal en el ámbito privado que no afecte a terceras personas, viola el derecho a la intimidad amparado por normas constitucionales. Actualmente se encuentra en el Congreso de la Nación un proyecto de reforma del Código Penal a ser tratado que, entre otras modificaciones, retoma este fallo con el fin de despenalizar la tenencia de estupefacientes que den cuenta de un consumo personal. No obstante, a la espera de este tratamiento, en el año 2017 se sancionó la Ley 27350 de Uso medicinal de la planta de Cannabis y sus derivados, la cual habilita el uso de la marihuana para tratamientos médicos, creando los ámbitos especializados para ello y permitiendo su uso como parte de tratamientos médicos. El Artículo 8 entra en conflicto con el Artículo 5 de la Ley 23737, al implementar la creación de un registro de los beneficiarios que accedan al uso del cannabis, con el fin de realizar una autorización que habilite el acceso y disipe lo estipulado en el Artículo 5 de la Ley 23737, el cual determina la penalización en casos de cultivo, comercialización y suministro o facilitar el estupefaciente.

El consumo de sustancias (especialmente, alcohol y tabaco) se encuentra entre los factores de riesgo más importantes en la región de las Américas para el desarrollo de enfermedades no transmisibles (cardiovasculares, respiratorias, cáncer y diabetes) ${ }^{(10)}$. En nuestro país, se estima que el $19,1 \%$ de las muertes en población de 15 a 65 años se relacionan de manera directa $(6,5 \%)$ y de manera indirecta $(93,5 \%)$ con el consumo de sustancias psicoactivas ${ }^{(11)}$. Los niveles más altos de consumo en territorio nacional se observan en jóvenes de 18 a 24 años. En un estudio realizado en 2017, en nuestro país, al tomar la prevalencia de año se observa en esta franja etaria que el $78,9 \%$ consume alcohol, el 33,5\% consume tabaco, el 15,9\% consume marihuana, el 3\% cocaína y el consumo de sustancias por vía inyectable es del $0,3 \%$. Además, se observa una baja percepción de riesgo respecto del consumo experimental (de una sola vez) u ocasional (algunas veces en los últimos 12 meses) de psicofármacos, marihuana, cocaína y también acerca de "fumar tabaco o marihuana" y "tomar alcohol de manera frecuente" (OAD, 2017). Los jóvenes de esta franja etaria son señalados como los que mayor riesgo presentan a la hora de consumir sustancias por su baja percepción de riesgo, por la alta prevalencia 
y por la modalidad de ingesta, en especial de alcohol $^{(2,12)}$. Con el fin de no seguir alimentando los estereotipos que existen sobre los jóvenes es importante aclarar que la mayoría no incurre en conductas de riesgo, si bien es la etapa de mayor vulnerabilidad (por ejemplo, con relación al consumo de sustancias) al ser comparada con otras etapas del ciclo vital $^{(13)}$.

De todas las sustancias psicoactivas, el alcohol representa la de mayor riesgo para este grupo etario en términos de morbimortalidad. En Argentina, la tasa de episodios de consumo excesivo aumentó del 2005 al 2010 en mujeres casi un 500\% y en varones casi un $100 \%^{(14)}$. Los estudios muestran que, entre los jóvenes, el consumo de sustancias (especialmente, alcohol, cannabis y cocaína) ha aumentado en los últimos años y, cuando la sustancia es el alcohol, cada vez se consume a edades más tempranas y de manera más riesgosa (grandes cantidades en lapsos breves $)^{(2,11,14)}$. De acuerdo con los registros de la Oficina de las Naciones Unidas contra la Droga y el Delito ${ }^{(15)}$, las nuevas drogas emergentes informadas se han cuadruplicado entre 2009 y 2015 incrementando su número de 166 a 644, lo que refleja una tendencia en franco crecimiento a nivel mundial.

En el presente estudio nos preguntamos respecto de las prácticas de cuidado y de riesgo que despliegan los jóvenes de entre 21 a 35 años de edad en escenarios nocturnos de presencia masiva de la Ciudad Autónoma de Buenos Aires: ¿cuáles son los riesgos que asumen o a los que quedan expuestos?, ¿Cómo se cuidan frente a estos riesgos asociados al ocio nocturno?, ¿se trata de prácticas individuales o de grupo?, ¿existen entornos más cuidados que otros?

La mayor parte de los estudios se ha centrado en los riesgos y unos pocos han indagado sobre las prácticas de cuidado asociadas al ocio nocturno. Entre los riesgos se destacan: el fácil acceso a las sustancias, la oferta constante, consumo excesivo y policonsumo, baja percepción de riesgo, tolerancia social, naturalización y permisividad sociofamiliar $^{(2,16,17)}$. Entre los jóvenes que asisten a bares y boliches en barrios vulnerabilizados surgen con mayor fuerza otros riesgos: la posibilidad de ser agredido o verse implicado en situaciones de violencia, ser expulsado del evento por el personal de seguridad y, en tercer lugar, una problemática referida exclusivamente por mujeres: el acoso o abuso sexual que pueden sufrir en estos escenarios ${ }^{(4)}$.

Respecto de las prácticas de cuidado, en un estudio con un grupo de jóvenes consumidores de drogas de diseño en la Ciudad de Buenos Aires se encontró que referían cuidarse informándose sobre los componentes de las pastillas y tomando recaudos para asumir menores efectos adversos, realizaban los consumos en contextos grupales, espacios y tiempos acotados y elegían este tipo de drogas por considerarlas más naturales, menos nocivas, más limpias, de fácil administración y poco adictivas ${ }^{(18)}$. En otro estudio realizado con jóvenes de contextos vulnerables y sectores medios que asistían a una variedad de escenarios nocturnos, se identificaron las prácticas de cuidado incorporadas, entre las que se destacaban: alimentarse adecuadamente antes de consumir, no consumir drogas si uno se encuentra atravesando problemas emocionales o afectivos, colocar de costado a quien consumió alcohol en forma abusiva para evitar que se ahogue con su propio vómito, tomar o darle mucha agua a quien consumió en exceso y salir/sacarlo al exterior, distribuir a lo largo de la noche las dosis y establecer horarios límite para el consumo, buscar información en sitios de Internet y blogs especializados en la temática ${ }^{(4)}$. Otro estudio llevado a cabo a fines de 2014, en una fiesta electrónica de la Ciudad de Buenos Aires, encontró que el $29 \%$ de los encuestados manifestó conocer su límite, el $23 \%$ expresó mantenerse hidratado, el $16 \%$ espaciar el consumo y el $8 \%$ ingerir algún alimento $^{(6)}$. La Global Drug Survey de 2017, que involucró a 115.523 personas $(68 \%$ sexo masculino, con una edad media de 29,1 años, más del $90 \%$ de etnicidad blanca, y más del $40 \%$ con título universitario) de 50 países -incluido Argentina- permitió conocer las estrategias y prácticas que empleaban los usuarios para lograr un consumo cuidado. Entre las de mayor frecuencia, mencionaron: 
dejar de consumir por unos días; comer antes de ingerir la sustancia; hidratarse muy bien durante la ingesta; dormir muy bien luego de consumir la sustancia; no excederse; evitar la ingesta de la sustancia durante el día; tener un dealer de confianza; conocer la calidad del producto; si es la primera vez, asesorarse con un usuario de la sustancia; planificar el consumo; no perder de vista a las amistades; evitar su uso cuando se está ansioso y/o deprimido; y evitar conducir vehículo o bicicleta $^{(19)}$.

Solo en el estudio de Güelman ${ }^{(4)}$ se destaca claramente el rol del grupo como lugar de cuidado. El grupo acompaña, procurando que no surjan problemas - derivados del consumo y de situaciones de violencia- o bien, liderando las estrategias de cuidado, especialmente de los consumidores novatos. Las prácticas de cuidado hacia el otro forman parte de un "contrato" implícito compartido entre todos y que implica "no dejar nunca a un amigo tirado". Aquellos jóvenes que asisten con frecuencia a fiestas electrónicas señalan que el cuidado y la contención del otro no se restringen a los integrantes del grupo de amigos, sino que puede extenderse a otros concurrentes.

Son escasos los estudios que han indagado de manera articulada las prácticas de riesgo y de cuidado y que han considerado en forma simultánea los tres niveles de análisis: individual, grupal y del entorno. El objetivo del presente estudio fue, por lo tanto, indagar las prácticas de riesgo y cuidado no solo a nivel individual sino grupal y del entorno, desde la percepción de los jóvenes que asiduamente concurren a escenarios nocturnos de presencia masiva de la Ciudad Autónoma de Buenos Aires. Asimismo, como objetivo secundario, se indagaron los motivos para consumir sustancias psicoactivas en estos escenarios. Por otra parte, el tipo de diseño empleado permitió identificar categorías conceptuales y establecer posibles relaciones estas, así como conocer cuáles son las prácticas de riesgo y de cuidado más destacadas a nivel individual, grupal y del entorno.

\section{METODOLOGÍA}

Se realizó un estudio con enfoque cualitativo, con el fin de comprender y profundizar el fenómeno del consumo de sustancias psicoactivas desde la perspectiva de los participantes en su ambiente natural y en relación con el contexto, los significados, las opiniones y las experiencias ${ }^{(20)}$. Se utilizó la técnica de entrevista semiestructurada a informantes claves y a través de un diseño basado en la teoría fundamentada ${ }^{(21)}$ se abordó el análisis de los datos, identificando los segmentos de sentido que mejor describen las prácticas de consumo de la población de referencia.

Participaron 16 jóvenes cuyas edades oscilaron mayormente entre 21 y 28 años (13 jóvenes, $81 \%$ ) y, el resto, entre 29 y 35 años (3 jóvenes, 19\%). La mitad eran mujeres. Los participantes fueron seleccionados por su frecuente concurrencia (más de una vez al mes) a escenarios nocturnos de presencia masiva (bares, pubs, fiestas electrónicas y boliches) de la Ciudad. Cinco de ellos, además de cumplir con este criterio, fueron seleccionados por su rol en este tipo de escenarios: relaciones públicas, barman, productor cultural o productor de programa en la noche porteña. Respecto del nivel de estudios alcanzado por las personas entrevistadas, tres tenían secundario completo; diez, universitario incompleto; una, terciario completo; y dos, universitario completo. Todos los participantes fueron comunicados sobre los alcances y objetivos del estudio tras lo cual prestaron su consentimiento informado según los principios éticos de igualdad, voluntariedad, anonimato, confidencialidad y seguridad $^{(22)}$. El proyecto contó con la aprobación del Comité de Ética para la Investigación Científica y Tecnológica de la Universidad Abierta Interamericana, código 0-1039.

Se elaboró una guía de preguntas con temas a tratar a partir de reuniones realizadas entre los investigadores. Los bloques temáticos resultantes fueron: lugar frecuentado, consumos (qué sustancias, con qué modalidad, etc.), riesgos y cuidados: ¿qué hacen cuando van a esos lugares?, ¿Cómo se cuidan?, ¿cuáles son los riesgos?, ¿por qué lo hacen? fueron 
algunas de las preguntas efectuadas. Al finalizar la entrevista se pidió a cada participante que represente en cinco palabras la noche de la Ciudad de Buenos Aires. La duración de cada entrevista fue de aproximadamente una hora. Todas las entrevistas fueron grabadas en formato de audio para facilitar la transcripción de la información y su posterior análisis. Los nombres reales fueron reemplazados por nombres ficticios para preservar el anonimato.

La información se volcó en una unidad hermenéutica en el programa informático Atlas.ti v.7.5.7 (2003-2018), utilizado como herramienta auxiliar. Se procedió a la lectura crítica de la información obtenida, la cual permitió generar categorías de primero y de segundo orden, analizadas exhaustivamente hasta su saturación con el objetivo de comprender los significados emergentes, de acuerdo con las diversas temáticas esenciales indagadas (cómo se cuidan, cuáles son los riesgos y por qué lo hacen) y siguiendo los lineamientos de la teoría fundamentada. Finalmente, se realizó la auditabilidad de las categorías obtenidas a través del procedimiento de doble ciego (dos grupos de investigadores analizaron todas las entrevistas de manera independiente para luego revisar las convergencias y discutir las divergencias hasta aunar criterios y lograr categorías consensuadas).

\section{RESULTADOS}

A continuación, se presentan las categorías de primer orden correspondientes a cada categoría del plano central, así como ejemplos de los segmentos de sentido que las fundamentan y permiten definirlas. Entre paréntesis, se presenta la cantidad de entrevistados (en total 16) que mencionaron segmentos de sentido correspondientes a esa categoría.

\section{Prácticas de riesgo individuales}

Ingesta excesiva (15): consumo excesivo en lapso breve o consumo sostenido durante varias horas.
Le dan mucho a la "previa" como para entrar súper entonados al boliche. $Y$ adentro, se quieren pasar. Yo veo que la gente quiere quebrar. Va directamente a quebrar. Tengo amigos y amigas o conocidos que dicen "yo quiero quebrar"... (Carlos, 23 años, relaciones públicas)

Se define "previa" al encuentro que se da antes de ingresar al evento y en el cual se suelen ingerir sustancias, generalmente alcohol en grandes cantidades, en un lapso breve ${ }^{(23)}$.

Mezclas (13): consumir sustancias sin considerar la combinación, el cómo combinar y cuándo hacerlo.

La gente mezcla mucho: mezcla alcohol, mezcla marihuana. Alcohol y marihuana siempre se mezcla. Cocaína también, en muchos de los casos se mezcla. Se mezcla... (Tomás, 24 años, asiste a boliches)

Me tomo una pasti y tomo más alcohol. Es más, si estoy medio volteado me tomo una pasti ["pastillas", drogas de síntesis] y vuelvo a estar arriba. Entonces puedo seguir tomando. Inclusive eso, no me afecta tanto el alcohol, no me doy cuenta y me puedo tomar diez whiskies... (Juan, 35 años, productor cultural)

Episodios violentos (9): Violencia, disturbio, peleas, asociados al desborde, sobreexcitación y al "no estar consciente" de la situación y que pueden darse tanto dentro como fuera del evento (muchas veces en la vía pública).

Siempre hay violencia. Casi siempre hay alguna. Pero a veces no lo podés evitar y hay conflicto igual. $Y$ si te tenés que pelear, te peleás. (Benjamín, 21 años, asiste a boliches)

Nunca faltan los que se agarran a trompadas [...] El alcohol los pone así. Saltan por cualquier cosa [...] La cosa parece que es pelearse, medirse y eso lo tienen que hacer tomados. (Sabrina, 25 años, asiste a pubs) 
Estar solo (4): Asistir a lugares sin amigos o haberse perdido de su grupo.

Algunos terminan en el piso porque les pegaron y los amigos se fueron. A los varones a veces los dejan solos en esas cosas. Las mujeres son más de estar acompañadas. (Sabrina, 25 años, asiste a pubs)

Desconocer la calidad del producto (4): Comprar sustancias psicoactivas a personas desconocidas, tomar lo que otros ofrecen, desconociendo la procedencia y calidad.

Yo aprendí a no comprar porque... una sola vez compré en un boliche y no me hizo nada. ¡Listo! Después lo que te vas enterando de que quizás las hacen muy fuerte o algo como pasó en la Time [refiere a la fiesta electrónica Ilamada Time Warp, en la que en 2016 fallecieron cinco jóvenes de entre 15 y 21 años y decenas debieron ser hospitalizadas] [...] Uno que sale, sabe que le vas a ir a comprar a un conocido que sabés que no te va a vender mierda. (Malena, 25 años, asiste a boliches)

Conducir vehículo (4): Manejar habiendo consumido sustancias psicoactivas.

He visto amigos subirse a las motos que prácticamente no las pueden mantener en pie [...] Siempre hay uno que se pasa un poco de copas y no es consciente. (Nicolás, 29 años, relaciones públicas)

\section{Prácticas de cuidado individuales}

Ingesta moderada (11): Conocer "el propio límite" referido al autocontrol respecto de la cantidad.

Si ya sabés que te estás pasando, aflojás un poco. (Benjamín, 21 años, asiste a boliches)
A veces se dan cuenta de que están abusando un poco y entonces cortan la noche. [...] Es llegar no al límite sino antes del límite. Pasarla bien, disfrutarlo y que sea una noche más. (Nicolás, 29 años, relaciones públicas)

Medidas de prevención (7): Preparar el cuerpo para la ingesta (hidratarse, alimentarse adecuadamente antes de consumir, etc.).

Se trata de comer antes. Estar hidratados. (Julieta, 24 años, relaciones públicas)

...gente conocida que frecuento tiene bastante claro cuáles son sus límites. Entonces saben qué consumen y qué no consumir cuando ya consumieron otra cosa. (Nicolás, 29 años, relaciones públicas)

Mezclas (5): Consumir una sola sustancia o conocer qué/cómo combinarlas.

No mezclás con alcohol. Todo lo que mezclan con alcohol, todo, llega un momento que no tiene un buen efecto, por así decirlo. (Malena, 25 años, asiste a boliches)

Información (3): Contar con información precisa acerca de los efectos combinados, conocer cuánto tiempo debe pasar entre dosis y dosis, etc.

Tipo dos de la mañana, te tomás la pasti, que dura hasta las seis, siete de la mañana [...] El alcohol se toma muchas horas antes de la pasti, en la "previa". (Lucía, 24 años, asiste a boliches)

El grupo de amistades o gente conocida que frecuento, la tienen bastante clara [...] Entonces saben qué consumen y qué no consumir cuando ya consumieron otra cosa. (Nicolás, 29 años, relaciones públicas)

Evitación de episodios violentos (2): Evitar situaciones potencialmente violentas. 
Porque pasa muchas veces que te empujan buscando lugar y se ponen molestos empujándote para atrás... todas esas cosas... Te corrés un poco, cedés la situación para no crear conflicto. (Benjamín, 21 años, asiste a boliches)

Calidad de sustancias (2): Conocer qué calidad de sustancia se está consumiendo, comprando en lugares o a personas de confianza.

Tenemos un amigo que él es el que se encarga de ir y buscarlas [pastillas]. Mayormente, antes de todo... de que, digamos, las reparta, las prueba él para saber si son buenas [...] Mayormente es a través de un conocido por un tema de que no sabés qué te pueden llegar a vender. (Malena, 25 años, asiste a boliches)

\section{Prácticas grupales de riesgo}

Consumo excesivo en/con el grupo (8): Señalan el consumo excesivo como actividad grupal, como vehículo del encuentro con el grupo de pares y como estrategia para pertenecer. Incluye "la previa" con consumo excesivo.

En "la previa", la gente toma mucho antes de salir porque le es más barato y para llegar más divertido también. Entonces, en ese momento se consume un montón... Entonces se juntan en las casas, en una esquina... Como que se consume antes de entrar a la fiesta porque en la fiesta es más caro. (Juan, 35 años, productor cultural)

Conductor designado (4): No tomar medidas para contar con un conductor designado o tener conductor designado que igual consume.

No tenemos conductor designado, sinceramente no... Eso está mal. Pero lo que pasa es que el que siempre maneja es mi novio y, la verdad es que ha manejado en distintos tipos de estados que, vos decís: "este pibe no puede manejar". Es más, creo que es más consciente estando drogado que borracho. (Malena, 25 años, asiste a boliches)

Presión del grupo (6): Percepción de presión por parte del grupo para que todos consuman.

Me joden mucho por eso [no consumo de alcohol] y medio que me quieren obligar a tomar. (Sabrina, 25 años, asiste a boliches)

\section{Prácticas grupales de cuidado}

Medidas de prevención grupal (12): Asistencia y contención por parte del grupo ("sacarlo a tomar aire", "pedir ayuda", "mojarle la nuca", etc.) y brindar información y consejo a otro miembro del grupo (qué mezclar y qué no, cuándo parar, tomar agua, etc.)

...Si el amigo está mal o muy borracho tirado siempre se queda uno cuidándolo, le van a buscar agua, tratan de que se recupere, lo llevan a su casa, en general en ese tema, sí. Por eso es importante salir acompañado. (Tomás, 24 años, asiste a boliches)

Entre las amistades se cuidan. Se ve mucho esto que te digo de que el que vas no quede tirado en la calle, que no se ahogue con el propio vómito, que no se lo lleve cualquiera. (Sabrina, 25 años, asiste a boliches)

Mantenerse en grupo (11): Mantenerse en grupo y no perder de vista a ningún miembro en ningún momento, es señalada como una estrategia clave.

Entramos todos juntos y tratamos de irnos todos juntos. A veces por distintos motivos algunos se van antes. Pero generalmente nunca se va medio solo, se van de a dos o a un par, pero nunca nadie solo. (Benjamín, 21 años, asiste a boliches)

En grupo, te sentís como más seguro. Si llega a pasar algo, o alguien se descom- 
pone, está toda la gente que está preocupándose por uno [...]. Entre todos nos cuidamos, nos ayudamos. (Julieta, 24 años, relaciones públicas)

Conductor designado (6): Cumplir con las condiciones de que el conductor designado no consuma sustancias o no llevar vehículo si todos quieren consumir.

Si salen con auto, no toman o se controlan mucho en la cantidad que toman. Por ahí, toman un poquito apenas entran al boliche: una cerveza u otra cosa y no toman en toda la noche. En ese sentido, sí se controlan. (Benjamín, 21 años, asiste a boliches)

\section{El entorno y sus riesgos}

Disponibilidad y oferta de sustancias (14): Refiere a la facilidad con que las sustancias psicoactivas pueden conseguirse y la alta frecuencia con que se oferta el consumo de sustancias psicoactivas por parte de asistentes al evento. Incluye sustancias de calidad dudosa.

Está el que va acompañado y que consume algo más que le pueden llegar a invitar ahí adentro del boliche o que lo pueda comprar adentro del boliche, como puede llegar a ser éxtasis o ketamina o cocaína. [...] Se consigue [...] hay gente que aprovecha esas fiestas para ir y vender dentro de esas mismas fiestas. (Tomás, 24 años, asiste a boliches)

...Si no sos firme con tu "no", un poco más te ponen en la cara, "Tomalo". [...] Si uno quiere conseguir, lo consigue. Está a la mano, es de fácil acceso. Dentro del boliche están como las personas que ya sabés que venden. Que capaz que están confabulados con alguien del boliche porque si no enseguida los sacarían. Vos capaz que vas caminando y te van diciendo "pasti", "keta", "LSD". (Julieta, 24 años, relaciones públicas)
Naturalización del consumo (12): Referido a la creencia o percepción de que es natural/ normal consumir algún tipo de sustancia ya que "todos lo hacen".

Yo creo que sobrio, por lo general -de la gente que he visto-, no he visto a nadie salir a un boliche y que esté sobrio, bajo efectos de ningún tipo de droga: la verdad que nunca lo vi. (Bárbara, 22 años, asiste a boliches)

Está muy naturalizada por todos lados, inclusive, no es algo que está legal, pero se fuma dentro de los boliches, se fuma en los bares, ya casi a la par del alcohol. Inclusive, yo creo que está como muy naturalizada socialmente, ahora cualquiera fuma porro. [...] Sin querer uno naturaliza mucho algo y ya no decide. (Juan, 35 años, productor cultural)

Fuerzas de seguridad (11): Abusos o desprotección por parte de las fuerzas de seguridad. "Patovicas", seguridad privada y policías que no controlan, que "hacen la vista gorda" habilitando la venta de sustancias y que reaccionan violentamente o expulsan a la persona del lugar dejándola expuesta a riesgos en la vía pública. Cuando se trata de mujeres, el riesgo de sufrir abusos por parte del personal de seguridad o de la policía y/o los riesgos en la vía pública son percibidos como sustancialmente mayores.

Los de seguridad te sacan del boliche $y$ chau. Te sacaron a la puerta, vos estás de la vereda para afuera, no sos problema de ellos y listo. Porque he visto que sacan a las chicas incluso por estar desmayadas o tiradas o algo así... O, por ejemplo, vomitando, que por ahí, Ilaman a la ambulancia y listo. Lo Ilevan ahí y no le hacen nada porque por ahí tenía solo alcohol, pero la echan porque estaba vomitando. Y la sacan a la calle y las tiran, porque sabemos que las maltratan, las sacan a la calle, las tiran y listo. Entonces como que no, no hay ayuda. (Bárbara, 22 años, asiste a boliches) 
Condiciones del lugar (11): Falta de cuidados por parte de los organizadores del evento al no controlar las condiciones del lugar como la capacidad ("falta de espacio", "permiten el ingreso de más personas que las que el lugar puede alojar") y al no cumplir con las normativas vigentes (como la de no fumar), falta de acceso al agua (por su alto costo o por cortar el suministro) y no tener asistencia médica.

Estás bailando y te sentís super apretado, te estás ahogando y te das cuenta que hay un montón de gente, vas al baño y no hay agua. (Lucía, 24 años, asiste a boliches)

Acoso/violencia de género (4): Abusos, acoso o agresión hacia persona de género mujer.

Las chicas están muy borrachas. Si no tienen amigos que las rescaten, la situación se pone fea. Porque los pibes están muy sacados y no miden [...] He visto que hayan echado a alguno por hacerse el vivo con alguna chica. Incluso me ha pasado. (Andrea, 22 años, asiste a boliches)

Acosos, por ejemplo, los flacos que salen, pasan un grupito de chicas y se ponen medios (sic) violentos con las palabras. (Carlos, 23 años, relaciones públicas)

\section{Entorno cuidado}

Fuerzas de seguridad (11): La contención y el control parten de las fuerzas de seguridad ("patovicas", seguridad privada y personal de policía cumpliendo rol de contención y de control, cuidando el cumplimiento de normas)

En los boliches que he estado yo, siempre la seguridad se ha hecho cargo del accidentado. O el chico que está pasando por algún problema, lo asisten enseguida. (Tomás 24 años, asiste a boliches)

Condiciones del lugar (8): Cuidados por parte de los organizadores hacia la gente que concurre, al controlar la capacidad del lugar (su- ficiente espacio), disponibilidad de agua (posibilidad de hidratarse), espacios y estrategias para contener y asistir a la persona que así lo requiere (servicio médico, VIP, disponibilidad de servicio de ambulancia, etc.).

No se llega a esa situación de ahogo porque está más controlado el tema de la capacidad permitida. (Andrea, 22 años, asiste a boliches)

Puede haber profesionales que se hagan cargo. En un festival importante, por ejemplo, tienen guardia y te asisten re bien. (Lucía, 24 años, asiste a boliches)

La regulación de estas condiciones es relativamente reciente, y surgió como resultado de antecedentes trágicos ocurridos en eventos nocturnos de CABA. Uno de ellos, la "tragedia de Cromañón" del 30 de diciembre de 2004 provocó una de las mayores catástrofes de este tipo en Argentina con un saldo de 194 muertos. Esta tragedia, puso al descubierto las serias fallas en la existencia y aplicación de medidas seguras en los diferentes escenarios (sea por encontrarse excedido en cantidad de personas, por el ingreso de pirotecnia, condiciones edilicias inseguras, etc.) y originó importantes cambios políticos y culturales. En lo político, dio lugar a la creación de un "Registro Público de Lugares Bailables", que implicó una reformulación de las condiciones de funcionamiento de los locales. El Decreto de Necesidad y Urgencia 1/2005 de la Ciudad de Buenos Aires establece, entre otros requisitos de habilitación y funcionamiento, la capacidad máxima permitida, plan de evacuación y materiales de revestimiento no combustibles ${ }^{(24)}$. A su vez, a nivel nacional, la Ley 26370 de Acceso a Boliches, del año 2008, establece la prohibición del acceso a establecimientos con pirotecnia $\mathrm{u}$ objetos que pongan en riesgo la seguridad de sí y de terceros allí dentro ${ }^{(25)}$. Además, impide el ingreso en caso de haberse encontrado cubierta la capacidad máxima del lugar. En el año 2010, el Decreto de Necesidad y Urgencia 3554 determinó la obligatoriedad del establecimiento tanto de informar en carteles externos cuál es su capacidad máxima como 
así también indicar si ya ha sido sobrepasada. Ayuda o consejo de gente desconocida (6): Asistencia y contención por parte de otras personas que aconsejan y ayudan a aquel que tiene alguna dificultad.

Yo me sentía re mal [...] vomité en el piso y me empezaron a tirar agua en el cuello. Ahí, como que reviví y me hicieron un poco más de espacio. Recuperé el aire... Listo, no pasó nada [...] Si te ven que estas mal o algo "¿che, no querés un caramelo?, ¿no querés un chicle?, ¿estás seguro?, ¿estás bien?, ¿no querés agua?" De la nada la gente se vuelve tu amiga y cuando vieron que vos estuviste bien, listo, dale, vamos a seguir bailando hasta las 6 de la mañana. (Malena, 25 años, asiste a boliches)

Códigos de convivencia (4): Rechazo de ciertas conductas (predominantemente en fiestas electrónicos) por parte de la mayoría de los asistentes, tal como episodio de violencia, cuestionando o excluyendo a quien lo genera, con el fin de conservar el "buen clima".

En las peleas uno trata de evitarlo, trata de evitar situaciones de conflicto. Como, no sé... No responder mal sin ir a buscar a la gente, insultarse, enojarse [...] Porque si se empiezan a empujar, siempre termina en lo mismo. (Benjamín, 21 años, asiste a boliches)

En estas fiestas, con el tiempo se hicieron códigos que, más o menos, se respetan... siempre hay un cuidado... el clima. (Lucía, 24 años, asiste a boliches)

Otro aspecto indagado refiere a los motivos para consumir que fueron agrupados en cuatro categorías (los números entre paréntesis hacen referencia a la cantidad de entrevistados que refirieron dicha experiencia).

Diversión (13): Implica despejarse, relajarse, desinhibirse y, a la vez, "desconectar" (de las obligaciones y la rutina).
[Se consume para] Charlar, bailar, divertirse, pasarla bien, distenderse de las tareas cotidianas [...] Distenderse, alejarse de la realidad. Estar en otro mambo, otra sintonía, y perderse, o sea, desinhibirse. (Nicolás, 29 años, relaciones públicas)

Encontrarse con otros (7): Implica "conectar" con otros, identificarse con el grupo, sensación de pertenencia y posibilidad de conocer gente (incluye encuentro sexual).

Hay algo que me gusta que tiene que ver con el encuentro, con la gente festejando, las cosas son para compartir y las drogas también. (Juan, 35 años, productor cultural)

Efecto farmacológico (8): Aumento de experiencia sensorial (sentir más, sentirse bien con uno y con todas las personas y las cosas).

Hay gente que dice "¡no, esta re bueno! te tomas un $L S D$, una pepa y te tomas un vodka, quedas re loco. Ves la música, escuchas los colores" [...] Es como que les gusta flashearla un poco más. (Carlos, 23 años, relaciones públicas)

Escape (7): Como estrategia para paliar la angustia y la ansiedad, olvidarse de los problemas y obligaciones de la vida diaria.
Liberar un poco la tensión de la semana o de los días, cada uno tiene sus propios problemas personales y para la mayoría de la gente es una salida para relajarse o distenderse, olvidarse de sus problemas. (Tomás, 24 años, asistente a boliches)

Finalmente, ante la solicitud de asociar cinco palabras que permitan definir la noche de Buenos Aires, 14 de los 16 entrevistados utilizó los términos diversión y alegría, poco más de la mitad (9) asoció la noche a drogas y alcohol, 7 a los amigos, 6 mencionaron los términos locura y descontrol y 6 , a la música. 


\section{DISCUSIÓN}

Conocer las prácticas de cuidado y de riesgo que se despliegan en escenarios nocturnos de presencia masiva es hoy una tarea básica necesaria para la planificación de acciones de prevención en territorio.

Investigaciones previas ${ }^{(1,3,4,6,7,9)}$, establecieron que en estos escenarios el consumo de sustancias cobra especial protagonismo como vehículo para la diversión, el encuentro con los otros y sentirse bien con uno mismo. En consonancia con estos resultados, en el presente estudio se encontró que las sustancias psicoactivas (incluido el alcohol) tienen una clara presencia en estos escenarios, fundamentalmente, como forma de aumentar la diversión y como vehículo del encuentro con otros. También se busca aumentar la experiencia sensorial a través de la ingesta de las distintas sustancias y, en última instancia, olvidar los problemas, las obligaciones y "romper" con la rutina.

Por otra parte, en el presente estudio, en un plano inicial, a partir de los segmentos de sentido se han establecido categorías de primer orden que pudieron ser ubicadas en torno a las categorías axiales (de segundo orden) de las cuales se habían partido: prácticas de riesgo y cuidado, individuales, grupales y del entorno.

Respecto de las prácticas individuales, el presente estudio replica los hallazgos de otras investigaciones ${ }^{(6,26)}$ respecto de los riesgos y cuidados en escenarios nocturnos. Saber qué consumir (conocer la calidad del producto), cuánto consumir ("conocer el propio límite", "no pasarse") y cómo consumir (cómo dosificar, hidratarse y alimentarse, si mezclar o cómo combinar las sustancias) son prácticas individuales que permiten minimizar los riesgos asociados al uso de drogas.

A nivel del grupo, los riesgos podrían clasificarse en tres categorías: consumo excesivo como práctica grupal (durante el evento y, muchas veces, antes, en el extendido formato de "previa"), falta de conductor designado y presión del grupo para que todos consuman. Pero a diferencia de lo que ocurre a nivel individual, a nivel del grupo los participantes del estudio destacan prácticas de cuidado. Mantenerse en grupo parece ser la clave. La frase "entre todos nos cuidamos" resuena con fuerza en las voces de los protagonistas del estudio y pareciera "hacer mecha" en cada encuentro nocturno como principal estrategia frente a posibles amenazas del entorno y ante eventuales riesgos individuales. Estar atentos y mantenerse unidos es una práctica altamente extendida, independientemente de los tipos de escenarios y de las edades de los participantes. La consigna "nunca nadie puede quedar solo" se traduce en norma grupal y si, por algún motivo, un miembro del grupo debe abandonar el lugar (por ejemplo, por haber sido expulsado del evento por parte de las fuerzas de seguridad), el grupo sacrifica la diversión de esa noche y acompaña a la persona para brindarle apoyo y contención. El grupo es también quien brinda los primeros auxilios cuando un miembro se descompone. Y solo acuden a la organización del evento o a la asistencia médica (cuando hay) como último recurso, cuando a pesar de las maniobras intentadas (hidratar a la persona, asegurar que tome aire, etc.) la persona sigue descompensada. El grupo deviene así el lugar en donde entre todos se cuidan, se brindan asistencia y contención. Por otra parte, los participantes advierten que las jóvenes son las que se encuentran expuestas a los mayores riesgos, por lo que estas prácticas de solidaridad y cuidado grupal se intensifican cuando quien necesita ayuda o debe abandonar el evento es una mujer.

En el entorno es donde se han señalado proporcionalmente mayores situaciones de riesgo. La alta disponibilidad y oferta de sustancias, es la categoría que surgió con más fuerza pese a la existencia de una legislación nacional que prohíbe el consumo, tenencia y venta de las sustancias (con excepción de alcohol y tabaco cuya prohibición se aplica solo a menores de 18 años). En segundo lugar, emergió la categoría denominada "naturalización del consumo" que opera como factor de riesgo, en tanto los jóvenes perciben que el consumo es lo esperable y hasta el 
abuso está justificado en estos entornos. Por otra parte, las condiciones del lugar fueron señaladas como otra fuente de riesgo cuando la normativa vigente que regula estos espacios no se cumple. Pero al mismo tiempo, reconocen que tales condiciones fueron mejorando a partir de las distintas tragedias que han tenido lugar en eventos nocturnos de Buenos Aires. Por otra parte, las fuerzas de seguridad son señaladas operando tanto a nivel de los riesgos como del cuidado. Ante ciertas situaciones ligadas muchas veces al efecto del consumo de sustancias, las fuerzas de seguridad ejercen un control represivo (los entrevistados hablaron de "abuso", "maltrato", "violencia" y "expulsión" del evento) u optan por no brindar asistencia ante una emergencia o situación de riesgo. Las situaciones de maltrato y expulsión se asociaron a una mayor percepción generalizada de riesgo cuando la persona involucrada es mujer. Si bien las fuerzas de seguridad fueron ubicadas como fuente de riesgos, los entrevistados también reconocen que, bajo ciertas circunstancias y en determinados escenarios, operan conteniendo y protegiendo de riesgos y potenciales daños (sin necesidad de acudir a estrategias violentas o de expulsión).

Respecto de la prevalencia de ciertas categorías sobre otras, a partir del análisis de la cantidad de entrevistados que mencionaron segmentos de sentido correspondientes a cada categoría, se puede concluir que en los escenarios nocturnos predominan las prácticas de riesgo, tanto individuales (sobre todo referidas a la modalidad de ingesta) como del entorno (referidas a las fuerzas de seguridad y condiciones del lugar). Sin embargo, a nivel del grupo se observa un predominio de prácticas grupales de cuidado por sobre las prácticas de riesgo. Los participantes del presente estudio coinciden en considerar que mantenerse en grupo y cuidarse entre todos es fundamental, así como brindar asistencia y contención a los miembros del grupo.

Históricamente, el grupo de pares ha sido señalado como uno de los factores de riesgo de consumo de sustancias. Se ha concluido que los jóvenes son particularmente sensibles a las recompensas cuando están en compañía de sus pares ${ }^{(27)}$ y son más propensos que los adultos a tomar riesgos en $\operatorname{grupo}^{(28,29,30)}$. En estudios realizados en los campus de universidades de EEUU, se encontró que el consumo excesivo de alcohol se asoció a los procesos de socialización que allí tienen lugar ${ }^{(31)}$ y que a mayor cantidad de alcohol consumida, mayor el estatus social (nivel de popularidad) alcanzado ${ }^{(32)}$. En otro estudio con población italiana ${ }^{(33)}$, se encontró que el consumo al interior de grupos de pares se asociaba a relaciones sociales valoradas de manera más positivas. En un estudio con población adolescente de Argentina se encontró que quienes más consumían alcohol reportaron mayor aceptación social, atractivo amoroso y autoestima que aquellos que consumían de manera moderada o no lo hacían $^{(34)}$. Y la decisión de consumir grandes cantidades de alcohol se asociaba a la necesidad de pertenencia a un grupo de pares ${ }^{(35)}$. En síntesis, los estudios han mostrado que la pertenencia a grupos de pares que consumen sustancias psicoactivas es un factor de riesgo para el inicio, mantenimiento y trayectorias problemáticas de consumo, y que el consumo de alcohol repercute de manera positiva en dominios de la vida social del joven. Referirse a los efectos que tiene pertenecer a un grupo de pares que consumen sustancias, no implica negar la evidencia que sostiene que por ciertas características de personalidad y actitud hacia el consumo, los jóvenes eligen determinado grupo de pares.

Entonces, ¿cuál es el rol del grupo en el espectro riesgo-cuidado? Efectivamente, el grupo de pares constituye una fuerte influencia social en las decisiones y el comportamiento que adoptan los jóvenes, y es un factor de riesgo en tanto ejerce presión a sus miembros que, de no aceptar sus normas y reglas respecto del consumo de drogas, pueden quedar excluidos. Pero en este trabajo se añade, además de la presión ejercida por el grupo que mencionaron los participantes, otra vertiente que pone en evidencia que la pertenencia opera como espacio de construcción de fuertes lazos sociales de solidaridad, hermandad y sororidad. En escenarios nocturnos, el grupo crea sus propios rituales, 
rutinas y sentidos en torno al consumo de sustancias y sus prácticas asociadas, pudiendo ejercer también influencias grupales facilitadoras para la construcción de apoyo colectivo. Por otro lado, a partir de las narraciones de los participantes, se infiere la aparición de vínculos solidarios in situ con capacidad de generar empatía por (y con) el otro y cooperación grupal ante la necesidad de auxilio que alguien requiera. Es por ello que resulta interesante y de gran importancia focalizar en estas fortalezas del grupo como una vía fértil para la ejecución de acciones de prevención y promoción de estrategias de cuidado en el entorno grupal y a nivel individual. El rol que ocupa el grupo como ámbito de cuidado resuena a potencialidad. En palabras de Zaldúa ${ }^{(36)}$ el grupo puede funcionar como potenciador de las transformaciones psicosociales en tanto logra construir tramas vinculares para el cuidado de sí y de los otros. En este sentido, los trabajos de Camarotti ${ }^{(1)}$ y Güelman ${ }^{(4)}$ destacan las fortalezas que construyen los jóvenes en torno a los riesgos asociados a la ingesta de sustancias psicoactivas, todo ello gracias a la participación en códigos grupales específicos.

El cuidado del otro transcurre en un contexto en el cual coexisten en simultáneo situaciones que representan sentidos antagónicos. Por un lado, se resalta el reservorio de solidaridad grupal reflejado en el cuidado que el grupo despliega frente a las situaciones riesgosas pero, por el otro, el grupo se encuentra inmerso en un contexto que, tal como fue expresado por los participantes del estudio, no constituye un lugar de refugio o cuidado plausible de acudir a él en caso de necesidad y, en muchas ocasiones, forma parte de los riesgos a los que se exponen cuando optan por esta forma de recreación. A través de la figura del personal de seguridad, se ofrecen como respuesta frecuente acciones de gran hostilidad manifestadas, ya sea mediante intervención directa con violencia o a través de la expulsión-abandono del joven, sometiéndolo a una mayor situación de vulnerabilidad al quedar expuesto a los riesgos de la vía pública.

En este punto, advertimos que no se trata ya de figuras o situaciones puntuales de violencia hacia los jóvenes en entornos nocturnos, sino de un medio sociocultural que, por un lado, ofrece constantemente sustancias y alienta su consumo y, por el otro, estigmatiza, condena y penaliza a los usuarios de drogas. La gran maquinaria mediática puesta al servicio de la construcción de una mirada prohibicionista y negativa con relación al consumo, se sostiene y se propaga socialmente mediante el magma de significaciones imaginarias ${ }^{(37)}$ que generan una imagen distorsionada creada mediante sentidos otorgados colectivamente, obstaculizando la construcción social de otras miradas posibles. En síntesis, la violencia es también sostener la estigmatización-patologización del joven que consume en una sociedad que, paradójicamente, ha entronizado el consumo de productos y bienes, no siempre necesarios, como vía para alcanzar el bienestar.

Finalmente, consideramos importante aclarar que resaltar la potencialidad grupal no implica des-responsabilizar a las políticas públicas ni dejar de interpelar el rol del Estado como actor fundamental con potestad de intervenir en estas temáticas. Los relatos de los sujetos de la presente investigación convergen en la presencia de respuestas del orden del desamparo y la violencia por parte del contexto ante situaciones de consumo, pero también interpelan a las políticas públicas, en tanto la prohibición y el control represivo no han logrado reducir el consumo ni evitar los riesgos asociados. Tampoco los programas y planes nacionales, dado los datos arrojados por los últimos estudios epidemiológicos en los que se advierte un aumento del consumo de algunas sustancias ${ }^{(2,11)}$, han logrado resultados positivos como consecuencia de las acciones implementadas.

En Argentina, existen planes, programas y proyectos destinados a la prevención primaria, secundaria y terciaria, y proyectos específicos a nivel nacional, provincial y municipal que se desarrollan desde hace años en centros educativos, centros comunitarios, organizaciones laborales y barrios vulnerables, entre otras iniciativas por fuera de la órbita gubernamental. Entre los más importantes se destacan: el Programa Nacional de Educación y Prevención sobre la Adicción y 
el Consumo Indebido de Drogas del Ministerio de Nación, creado por el Ministerio de Educación y convertido en Ley 26586, en el año $2009^{(38)}$, la Ley 26934 Plan Integral de Abordaje de Consumos Problemáticos promulgado en el año 2014 por el Poder Ejecutivo ${ }^{(39)}$, el reciente Proyecto "ESTAR" en Prevención de la Dirección Nacional del Observatorio Argentino de Drogas, creado en 2018, que propone acompañar a las comunidades educativas y sus actores ante situaciones previamente detectadas de consumo problemático, el proyecto Cuidados en Juego de la Secretaría de Programación para la Prevención de la Drogadicción y la Lucha contra el Narcotráfico, creado en 2017, que provee materiales didácticos destinados a trabajar la prevención de los consumos problemáticos en los diferentes niveles del sistema educativo.

Las políticas públicas debieran recuperar su rol estratégico con acciones tendientes al alcance de un estado saludable en la población y garantía de derechos ${ }^{(40)}$, con programas que adhieran a los lineamientos de los enfoques estratégico-participativos (no normativos y verticalistas $)^{(41)}$ y desde un enfoque ideológico que priorice la reducción del riesgo asociado al consumo y desestigmatice la sustancia, el consumo y a los consumidores ${ }^{(42)}$.

\section{Implicancias prácticas}

Conocer las prácticas de cuidado grupales invita a potenciarlas desde un lugar no normativo, no reduccionista y no estigmatizante de la cultura juvenil y sus sentidos, sino desde un enfoque estratégico-participativo que considera las voces de los jóvenes, sus fortalezas y su potencial transformador ${ }^{(13)}$. Algunas iniciativas desde la perspectiva de reducción de daños han ido en este sentido y merecen ser destacadas. El enfoque de reducción de daños promueve un conjunto de ideas y estrategias orientadas ya no a abolir o eliminar la conducta-problema sino a reducir las consecuencias negativas asociadas. Surgida en los inicios de la década de 1980, esta concepción tiene como principal objetivo minimizar los efectos negativos asociados al consumo de sustancias psicoactivas, sin que esto implique restarle importancia a los riesgos y daños por el consumo ${ }^{(42)}$.

Este enfoque contempla la acción civil de la población organizada motu proprio en asociaciones no gubernamentales sin fines de lucro que en latitudes europeas se agrupan en torno a "Energy Control" con sede en España y en Sudamérica, en "Échele Cabeza Cuando se dé en la Cabeza" con asentamiento en Colombia. Dentro de sus actividades, se realizan intervenciones in situ en eventos masivos, tanto en la provisión de asistencia, de promoción de la salud y de difusión de prácticas de cuidado como también información fehaciente sobre la base de testeo de sustancias psicoactivas, en virtud de la protección de la legislación vigente en sendos países. En nuestro contexto local, existe desde el 2016, el Proyecto de Atención en Fiestas "PAF, un cachetazo al mal viaje" mediante el cual la Asociación Civil Intercambios impulsa la formación de un equipo de jóvenes voluntarios que asisten a fiestas para implementar estrategias de intervención que promuevan prácticas de cuidado y consumo responsable entre usuarios de sustancias psicoactivas con fines recreativos.

\section{Limitaciones y perspectivas futuras}

Este estudio permitió conocer los riesgos y cuidados percibidos por el grupo de jóvenes entrevistados. Una serie de limitaciones deben ser mencionadas. La primera se refiere a que, debido a que se trata de la percepción que ellos tienen de lo que ocurre en entornos nocturnos, no sabemos si efectivamente estas prácticas ocurren en el escenario natural y con qué frecuencia. Por ejemplo, para los jóvenes entrevistados es bien conocida la figura del conductor designado. Sin embargo, a partir de este estudio, no es posible conocer en qué medida se recurre a ella. Por otra parte, una mayor variabilidad de jóvenes entrevistados puede contribuir a lograr otras miradas y sentidos que pudieron haber quedado subvalorados o desestimados en el 
presente estudio. Resta además conocer en qué medida estos riesgos y cuidados varían en función de los distintos tipos de escenarios. Cuestiones de género fueron sucintamente mencionadas, pero sería interesante indagar con mayor profundidad esta temática en futuros estudios. También la edad de los participantes en eventos nocturnos, según los informantes claves, tiene algún impacto en las prácticas que se adoptan. Nuevamente, fue escasamente indagada esta temática en el presente estudio. En investigaciones futuras, podría profundizarse también en los sentidos que los protagonistas dan a términos tales como "violencia", "ayudar", "conectar", etc. ya que podrían no tener el mismo significado para todos. En un próximo estudio se indagará, a partir de la aplicación de una encuesta a un grupo amplio de jóvenes, en qué medida ocurren las prácticas de cuidado y de riesgo, considerando de manera desagregada el género, la edad, el tipo de escenario y las sustancias que suelen consumir. De este modo, se contará con evidencia que podría contribuir a acciones de prevención que atiendan a las particularidades de cada escenario y resultar, por ende, en intervenciones más eficaces.

Finalmente, consideramos fundamental escuchar las voces de los jóvenes y conocer sus estrategias de cuidado, ya que representan la posibilidad de expandirlas y multiplicarlas, superando el paradigma de la responsabilidad individual y virando el eje hacia la responsabilidad colectiva en tanto el consumo es una práctica grupal en un contexto social determinado.

\section{AGRADECIMIENTOS}

A los jóvenes que participaron de la presente investigación. A los organismos que financiaron el proyecto: Consejo Nacional de Investigaciones Científicas y Técnicas (CONICET), Universidad Abierta Interamericana (UAI), Universidad de Buenos Aires (UBA).

\section{REFERENCIAS BIBLIOGRÁFICAS}

1. Camarotti AM. Individualmente juntos: Tensiones en las identidades juveniles en torno al consumo de éxtasis y la cultura dance. Apuntes de Investigación del CECYP. 2014;24(1):81-117.

2. Observatorio Argentino de Drogas. Estudio Nacional en población de 12 a 65 años, sobre consumo de sustancias psicoactivas: Argentina 2017. Buenos Aires: Sedronar; 2017.

3. Sánchez Antelo V, Mendes Diz A. Prácticas y sentidos de los riesgos: el autocuidado en los consumidores de drogas. Argumentos: revista de crítica social. 2015;17:357-386.

4. Güelman M. Entre nosotros nos cuidamos siempre: consumos de drogas y prácticas de cuidado en espacios recreativos nocturnos. En: Di Leo P, Camarotti A, (comp.). Individuación y reconoci- miento: Experiencia de jóvenes en la sociedad actual. Buenos Aires: Teseo; 2015.

5. Agencia Gubernamental de Control. Registro Público de Lugares Bailables. Buenos Aires: Ministerio de Justicia y Seguridad; 2019.

6. Observatorio Argentino de Drogas. Estudio exploratorio sobre el consumo de sustancias psicoactivas en fiestas electrónicas. Buenos Aires: Sedronar; 2016.

7. Oleaque J. En éxtasis: El bakalao como contracultura en España. Valencia: Barlin Libros; 2017.

8. Cámara Argentina de Cerveza Artesanal. Informe de la cámara de cerveceros argentinos. Buenos Aires: CAPCA; 2018.

9. Camarotti A, Kornblit A. Abordaje integral comunitario de los consumos problemáticos de drogas: construyendo un modelo. Salud Colectiva. 2015;11(2):211-221.

10. Organización Panamericana de la Salud. Estado de Salud de la población. Prevención y control de las enfermedades no transmisibles [Internet]. 2017 [citado 10 dic 2018]. Disponible en: https://tinyurl. com/y3yeeuv8.

11. Observatorio Argentino de Drogas. Mortalidad relacionada con el consumo de sustancias psicoactivas: Argentina 2017. Buenos Aires: Sedronar; 2019. 
12. Observatorio Interamericano sobre Drogas. Informe sobre el consumo de drogas en las Américas. Washington DC: CICAD-OEA; 2019.

13. Schmidt V, Giménez M, Cano V, Tamay ME, Cataldi S, González MA, Raimundi MJ, Vargas Y, Bugallo L, Donatti S. Evaluación psicológica en el ámbito social-comunitario: De la teoría clásica de la medición hacia la evaluación al servicio de la transformación social. Buenos Aires: Eudeba; 2016.

14. Organización Panamericana de la Salud. Regional status report on alcohol and health in the Americas. Washington DC: OPS; 2015.

15. Oficina de las Naciones Unidas contra la Droga y el Delito. Informe Mundial sobre las drogas 2017. Viena: Naciones Unidas; 2017.

16. Carreter Parreño J, García Castillo O, Ródenas Aguilar JL, Gómez Saldaña A, Bermejo Cacharrón Y, Villar Garrido I. Estudio cualitativo sobre tóxicos en adolescentes. Atención Primaria. 2011;43(8):435-439.

17. Castaño Ruiz V. Diferencias en la percepción de consumo recreativo de drogas entre chicos y chicas jóvenes: Un análisis desde la perspectiva de género. Madrid: Fundación Atenea; 2014.

18. Camarotti AM. Experiencias comunitarias de cuidado y diversión en circuitos de música electrónica. En: Di Leo P, Camarotti A, (comp.). Individuación y reconocimiento: Experiencia de jóvenes en la sociedad actual. Buenos Aires: Teseo; 2015.

19. Winstock A, Barrat M, Ferris J, Maier L. (2017). Global Drug Survey GDS 2017: Key findings report [Internet]. 2017 [citado 10 dic 2018]. Disponible en: https://tinyurl.com/y6f775bz.

20. Hernández Sampieri R, Fernández-Collado C, Baptista Lucio P. Metodología de la investigación. 4ta ed. México: McGraw-Hill Interamericana; 2014.

21. Strauss A, Corbin J. Bases de la investigación cualitativa: Técnicas y procedimientos para desarrollar la teoría fundamentada. Medellín: Editorial Universidad de Antioquia; 2002.

22. Organizaciones Internacionales de las Ciencias Médicas. Pautas éticas internacionales para la investigación relacionada con la salud con seres humanos [Internet] 2017 [citado 10 dic 2018]. Disponible en: https://tinyurl.com/y2ksfkuz.

23. Dirección Nacional de Salud Mental y Adicciones. Lineamientos para la atención del consumo episódico excesivo de alcohol en adolescentes. Buenos Aires: Ministerio de Salud de la Nación; 2018.
24. Gobierno de la Ciudad Autónoma de Buenos Aires. Decreto de Necesidad y Urgencia 1/ GCABA/05 [Internet]. 2005 [citado 10 dic 2018]. Disponible en: https://tinyurl.com/y5h5vwfs.

25. Argentina. Ley 26370, Espectáculos públicos [Internet]. 26 may 2008 [citada 10 dic 2018]. Disponible en: https://tinyurl.com/y6dpawd2.

26. Faura F, García N. El ocio nocturno y la reducción de riesgos. En: Martínez Oró P, Pallares Gómez N. De riesgos y placeres: Manual para entender las drogas. Lleida: Milenio; 2013.

27. Silva K, Shulman EP, Chein J, Steinberg L. Peers increase late adolescents' exploratory behavior and sensitivity to positive and negative feedback. Journal of Research on Adolescence. 2016;26(4):696-705. doi:10.1111/jora.12219.

28. Gardner M, Steinberg L. Peer influence on risk taking, risk preference, and risky decision making in adolescence and adulthood: an experimental study. Developmental Psychology. 2005;41(4):625-635. doi: 10.1037/0012-1649.41.4.625.

29. Smith AR, Rosenbaum GM, Botdorf MA, Steinberg L, Chein J. Peers influence adolescent reward processing, but not response inhibition. Cognitive, Affective, \& Behavioral Neuroscience. 2018;18(2):284-295.

30. Steinberg L, Monahan K. Age differences in resistance to peer influence. Developmental Psychology. 2007;43(6):1531-1543. doi: 10.1037/ 0012-1649.43.6.1531.

31. Lorant V, Nicaise P, Soto VE, d'Hoore W. Alcohol drinking among college students: college responsibility for personal troubles. BMC Public Health. 2013;13:615-624.

32. Dumas TM, Graham K, Bernards S, Wells S. Drinking to reach the top: Young adults' drinking patterns as a predictor of status within natural drinking groups. Addictive Behaviors, 2014;39:1510-1515.

33. Tartaglia S. Alcohol consumption among young adults in Italy: The interplay of individual and social factors. Drugs: Education, Prevention and Policy. 2014;21(1):65-71.

34. Calero A, Schmidt V, Bugallo L. Consumo de alcohol y su relación con la autopercepción adolescente: Alcohol consumption and its relationship with the adolescent self-perception. Health and Addictions. 2016;16(1):49-58.

35. Leibovich N, Schmidt V, Calero A. The need to belong (NB) in adolescence: Adaptation of a scale for its assessment. Psychology and Behavioral 
Science International Journal. 2018;8(5):555747. doi: 10.19080/PBSIJ.2018.08.555747.

36. Zaldúa G. Territorios, políticas sociales y actores: intervenciones preventivas y promocionales en salud. Buenos Aires: Teseo; 2016.

37. Castoriadis C. Lo imaginario: la creación en el dominio socio-histórico. Barcelona: Gedisa; 1988.

38. Argentina. Ley 26586 Programa Nacional de Educación y Prevención sobre las Adicciones y el Consumo Indebido de Drogas [Internet]. 30 dic 2009 [citado 10 dic 2018]. Disponible en: https:// tinyurl.com/y5e9w53y.

39. Argentina. Ley 26934 Plan Integral para el Abordaje de los Consumos Problemáticos [Inter- net]. 28 may 2014 [citado 10 dic 2018]. Disponible en: https://tinyurl.com/gpaenjw.

40. Argentina. Ley 26657 Derecho a la Protección de la Salud Mental [Internet]. 2 dic 2010 [citado 10 dic 2018]. Disponible en: https://tinyurl.com/ zl7bzbl.

41. Amaya P. El Estado y las políticas públicas en América Latina. La Plata: Universitaria de La Plata; 2010.

42. Damin C, Arrieta E. "¿Qué es la reducción de riesgos y de daños?". En: Un libro sobre drogas. Buenos Aires: El Gato y la caja; 2017.

\section{FORMA DE CITAR}

Schmidt V, Martucci F, Di Puglia G, Lo Giusto O, Rijana I, Alvarez Iturain A. Estudio cualitativo sobre prácticas de riesgo y de cuidado en escenarios nocturnos de presencia masiva de la Ciudad Autónoma de Buenos Aires. Salud Colectiva. 2019;15:e2171. doi: 10.18294/sc.2019.2171.

Recibido: 8 de febrero de 2019 | Versión final: 18 de julio de 2019 | Aprobado: 30 de agosto de 2019

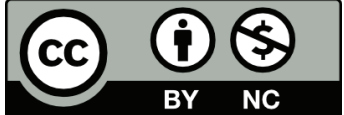

Esta obra está bajo una licencia de Creative Commons Reconocimiento-NoComercial 4.0 Internacional. Reconocimiento - Permite copiar, distribuir y comunicar públicamente la obra. A cambio, se debe reconocer y citar al autor original. No Comercial - Esta obra no puede ser utilizada con finalidades comerciales, a menos que se obtenga el permiso. 